\title{
William Wilkie Collins: la medicina como un medio para enriquecer la creación de la literatura de ficción
}

\author{
MARCELO MIRANDA C.
}

\section{William Wilkie Collins: Medicine as an aid for enriching the creation of fiction literature}

William Wilkie Collins (WWC) is a well-known prolific and innovator English writer of the Victorian age. After 150 years he is still being extensively read and all his main works are translated into Spanish. Furthermore, WWC has been acclaimed by famous writers such as JL Borges and TS Eliot as one of the best story-tellers of all times, and a master of the complexities of the plot of stories. WWC mentioned on his works many innovator aspects of medicine, demonstrating an uncanny power of observation and real interest in science. Notoriously, WWC described posttraumatic epilepsy, the clinical effects of opium, the introduction of people with sensorial deficits, such as blindness and deafness, as main figures in his novels. WWC also showed an interest in the management of mental disorders and the use of music as a potential therapy. In this review, we comment these interesting aspects of the creative work of this genius of the fiction literature.

(Rev Med Chile 2011; 139: 1365-1369).

Key words: Medicine in literature; Neurosciences; Opium.

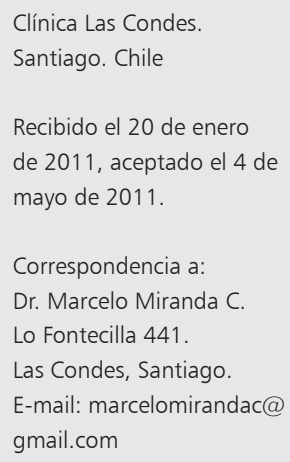

"El médico que no es lo suficientemente honesto para confesar cuando está desconcertado es un miembro habitual de la profesión médica en todos los países".

Wilkie Collins en "La Hija de Jezabel” (1880)

"En cualquier parte que en la trama de la obra se formulen preguntas acerca de la Ley, Medicina y la Química, la obra ha sido enviada, antes de su publicación, al juicio de individuos profesionales".

Wilkie Collins en "Armadale” (1868)

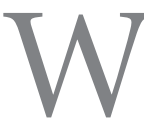
illiam Wilkie Collins (WWC)(18241889) (Figura 1) es un prolífico e innovador escritor de la época victoriana, con plena vigencia: la totalidad de su obra está traducida y es apreciada aún después de 150 años ${ }^{1}$. Contemporáneo de Charles Dickens con el cual co-escribió diversas obras y fue compañero de actuación en la representación teatral en otras, llegó en un momento a ser más valorado y mejor remunerado que su colega ${ }^{2,3}$. WWC logró además relevancia internacional al ser reconocido por los escritores y críticos de la talla de Jorge Luis Borges y T.S. Eliot ${ }^{4,5}$, como uno de los grandes narradores del siglo XIX y junto a Poe, el padre de la novela policial y de misterio. Sus novelas "La piedra lunar" (Figura 2) y "La dama vestida de blanco" están consideradas entre las mejores obras de la literatura universal ${ }^{4,5}$. En aspectos literarios, su obra fue innovadora también por introducir por primera vez la narración a través de múltiples personajes en la obra.

WWC, sin tener ningún tipo de formación médica, ya que tuvo básicamente estudios de derecho sin ejercer, presentó en numerosas obras aspectos médicos novedosos para su época con gran detalle, revelando un extraordinario poder de observación e interés en aspectos científicos ${ }^{6,7}$.

En esta revisión destacamos esos novedosos 


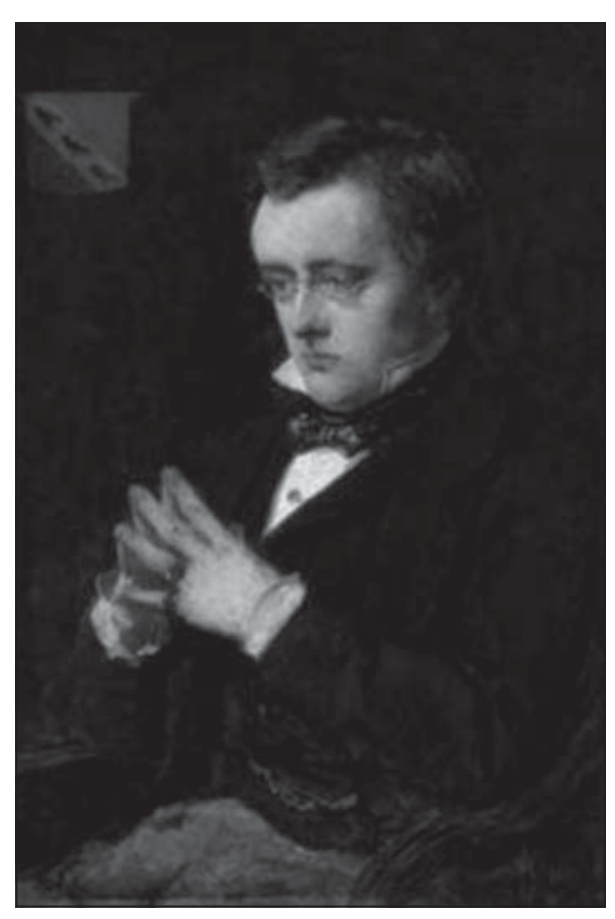

Figura 1. Wilkie Collins en un retrato de Millais en la época de mayor éxito a raíz de la novela "La Dama Vestida de Blanco" (1860)2.

aspectos médicos aún poco conocidos en la actualidad, si bien en nuestro país WWC es un escritor bien difundido.

\section{Epilepsia y su tratamiento}

En su novela "Pobre señorita Finch" (1872), WWC claramente describe en uno de los protagonistas de la obra, Oscar, crisis epilépticas de origen post traumático y su tratamiento con nitrato de plata ${ }^{7,8}$. WWC llega a describir el efecto adverso conocido de esta antigua terapia, como es la pigmentación obscura de la piel ${ }^{7}$. En esa época era tal la frecuencia del uso del nitrato de plata, que era común encontrar, como dice WWC en la obra, cientos de personas con esa coloración de piel, sin embargo, el tratamiento sobre la epilepsia era inefectivo ${ }^{8}$. WWC describe con sorprendente detalle una crisis versiva al lado derecho, es decir, giro de la cabeza a derecha seguido de crisis tónicoclónico generalizadas:

"una grotesca mueca invadió a Oscar, giró su cabeza a derecha, y luego sus ojos hacia arriba, cayó al suelo con un convulsión de todo su cuerpo. El Dr

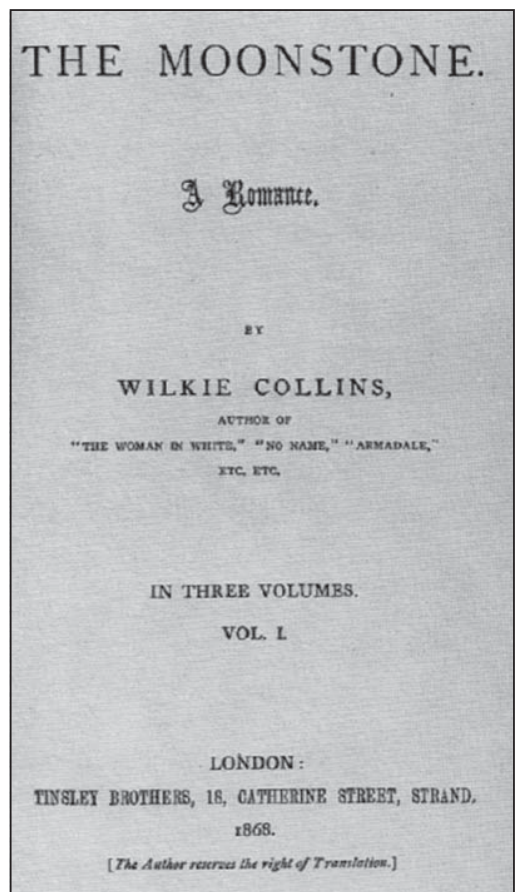

Figura 2. La primera edición de "La piedra Lunar" (1868)².

retiró algunos muebles para evitar que se golpeara, $y$ le pregunté ¿Por Dios qué es ésto? ¿Qué se puede hacer?' El Dr. movió negativamente la cabeza y dijo: nada, es una crisis epiléptica"s.

Es llamativo que ni el defecto visual de la joven Finch protagonista de la obra, ni la epilepsia de Oscar, son vistos con una connotación negativa, al contrario, provocan apoyo y consuelo de sus amigos y son un incentivo en ambos casos para superar satisfactoriamente la enfermedad e insertarse bien en la sociedad.

No tenemos noción de una descripción similar en la literatura no médica de esos años, salvo la obra contemporánea de Dostoievski, "El Idiota"7.

\section{Efectos del opio y su dependencia. \\ Una de las primeras novelas con una base "psicofarmacológica"}

WWC sufrió de una aparente gota muy invalidante, para lo cual se autoprescribió opio en forma de láudano ${ }^{2,3}$. El láudano se produce de una mezcla de opio con alcohol y es así ingerido, su empleo 
data de los tiempos de Paracelso. WWC llegó a usar dosis muy altas y experimentar sus efectos secundarios y tal como lo mencionaron, con su propia experiencia, su compatriota Thomas de Quincey, y el francés Charles Baudelaire, aprovechó estas manifestaciones para enriquecer sus obras $^{1,9}$. Por ejemplo, en una de novelas maestras, "La piedra lunar" (1868), uno de los personajes, R. Blake, preocupado por la seguridad de un famoso brillante que su prima recibe como herencia, joya adorada por los hindúes y usurpada por los ingleses, bajo los efectos del láudano indicado para una noche de insomnio, inconscientemente traslada el diamante a otro lugar que nadie logra determinar. Un año después, un asistente del Dr. Candy, quien había recetado la droga, se da cuenta que el efecto del laúdano pudo haber sido el causante de este acto y le solicita al afectado repetir la experiencia volviendo a ingerir láudano ${ }^{10}$. Esto está basado en el conocimiento extraído de literatura científica de la época, en que se sugería que una segunda ingesta no se asociaba a un claro trastorno de conciencia, pero si a repetición de los actos motores ${ }^{11}$. Al producirse el mismo acto de automatismo del año anterior, se logra determinar dónde se hallaba el diamante desaparecido. Este efecto conocido como disociación por drogas no fue descrito oficialmente hasta 1937 por un grupo de fisiólogos. WWC afirmó que utilizó este recurso al informarse con distintos médicos de los potenciales efectos del láudano, además de su propia experiencia. WWC claramente conocía los efectos psicológicos de su dependencia y el trastorno de conciencia que podía provocar. Reconoció además que parte de los capítulos de esta obra, fueron escritos bajo los efectos de la droga e incluso no recordaba cómo había logrado escribirlos ${ }^{1}$.

WWC sufrió de una dolorosa artritis y compromiso ocular que se atribuyó a una gota, si bien parece corresponder más bien a manifestaciones de lo que ahora denominaríamos probablemente una artritis seronegativa ${ }^{1-3}$. Llegó a ingerir grandes cantidades de opio, y según los doctores de la época, lo suficiente para matar a toda una tripulación. De hecho un criado de WWC involuntariamente bebió la mitad de un vaso de láudano, bastante menos que lo que WWC ingería a diario, lo que le causó la muerte instantáneamente ${ }^{1}$. Uno de sus personajes de la novela "Armadale" (1866), Lydia Gwilt dice: "bendito sea quien inventó el opio, le doy las gracias desde el fondo de mi corazón”12. En esta misma obra, la pérfida protagonista utiliza una extraña mezcla de láudano y otros químicos, originando un gas venenoso incoloro e inodoro con el cual asesina a otro de los protagonistas ${ }^{12}$. WWC reconoce hacer estado presente en la elaboración de un producto similar junto a un químico ${ }^{12}$.

WWC refiere alucinaciones provocadas por el constante y exagerado uso de láudano: "siento a alguien que está a mi lado y me acompaña a dormir; además en mi dormitorio se me aparece una mujer vestida de verde"1.

Para muchos críticos los últimos 15 años de la productividad literaria de WWC muestran logros menos exitosos y esto estaría reflejando las consecuencias del prolongado abuso del opio ${ }^{1-3}$. WWC murió el año 1889 como consecuencia de un accidente vascular encefálico.

\section{Los defectos sensoriales sordomudez y ceguera como tema central de algunas obras.}

Según nuestro conocimiento, por primera vez en la historia de la literatura, WWC coloca como protagonistas a personajes que padecen defectos sensoriales como sordomudez y ceguera. En particular WWC resalta cómo estos personajes se sobreponen a sus defectos, provocan simpatía y no rechazo de sus cercanos, cambiando así la impresión que tenemos que en esa época estas personas eran aisladas y no valoradas.

En el personaje Mary, de "El juego del escondite" $(1854)^{13}$, quien es sorda luego de un trauma craneal, WWC describe en detalle el examen realizado por diversos médicos, incluyendo el uso de otoscopia junto a una variante del test que conocemos en la actualidad como test de Weber. El test consiste en aplicar un diapasón por vía ósea (frente, huesos nasales o incisivos superiores). Lo normal es que el sonido sea escuchado al centro de la cabeza o en los dos oídos simultáneamente, lo que se denomina que el "Weber no lateraliza". Si estamos frente a un paciente con hipoacusia en un determinado oído y se aplica el diapasón en la frente, éste se oirá primero o lateralizará en el oído sano en el caso de que la hipoacusia sea de tipo sensorial o neural, ya que el contacto óseo no logra estimular el nervio dañado en este caso. Si la hipoacusia es de tipo conductiva, el Weber lateralizará hacia el lado enfermo (la vibración se transmite directamente al nervio indemne por el 
contacto del vibrador con el hueso). En el libro al protagonista se le coloca un reloj dentro de la boca ( simula el contacto óseo), y se le pide a través de un mensaje escrito decir si escucha el tic-tac del reloj. En este caso no lo escuchaba por lo que el médico acertadamente determinó un daño neural y por ende la irreversibilidad de la hipoacusia ${ }^{13}$. Es sorprendente la claridad y precisión como WWC describe el examen como si fuera él mismo quien examinara a la paciente.

Una evaluación médica similar en lo exhaustiva, ocurre con la protagonista de "Pobre señorita Finch", quien tiene una ceguera por cataratas y es reevaluada por el Dr. Gosse, según la historia, un conocido oftalmólogo alemán de la época. Fue operada sin éxito, con intención de reestablecer la visión. La descripción tan real del médico oftalmólogo llevó a cientos de lectores, a pesar de la fallida intervención, a pedir a WWC la información de cómo ubicar al Dr. Gosse ${ }^{3}$.

La estrategia para tratar trastornos nerviosos: inicios de la psicoterapia y musicoterapia? Otros avances técnicos no-médicos.

En su obra Armadale (1868), WWC pone en boca de un médico a cargo de un sanatorio de "enfermedades nerviosas", lo siguiente: " ¿cual es el tratamiento de la ansiedad mental que aflige a alguien y que hace que este alguien acuda a un médico?. Él le examina, le escucha y le prescribe 2 cosas. Una de ellas la escribe en un papel y es enviada a un farmacéutico. La otra se administra verbalmente, en el momento adecuado y consiste en la recomendación general de mantener tranquila la mente. En mi sanatorio en cada habitación disponemos de un sistema de purificación de aire, entregando un aire fresco y además con sólo un botón podemos abrir y cerrar puertas y ventanas sin provocarle ruido al paciente, ayudando así a mantener su tranquilidad. Además nos ayudamos de música como la de Haendel y $\mathrm{Ha}$ $y d n$ para así contribuir a relajarlos y darles paz"12.

Es muy interesante lo que afirma WWC en su personaje, porque en nuestra opinión, está haciendo alusión a esbozos de lo que conocemos hoy como psicoterapia, algunos años antes que Charcot, Freud y otros psiquiatras delinearan esta forma de terapia. WWC a través de su personaje también hace mención al empleo pionero de la música como herramienta terapéutica.

En la parte práctica, hizo referencia quizás a un mecanismo precursor de sistemas de purificación de aire $\mathrm{o}$ aire acondicionado y de sistemas eléctricos de cierre de ventanas y puertas, en una línea similar a las predicciones de Jules Verne.

\section{Discusión}

Si bien WWC tiene fama de escritor de misterio y novelas policíacas, lo que puede para algunos parecer peyorativo y superficial, su aporte literario conlleva mucho más que esas características de su creación, tal como sucedió con Edgar Allan Poe. En muchas de las obras de WWC, a diferencia de Dickens por ejemplo, quien hace referencia quizás en forma más superficial a aspectos médicos, la medicina adquiere un rol muy importante en la trama. No tenemos conocimiento de otro escritor de ficción de esa época, con excepción de H.G.Wells, que haya hecho tal número de interesantes y bien documentados aportes científicos en su creación literaria. En cada obra en las que incluyó temas médicos, WWC comentó en los prólogos o apéndices cómo obtuvo esa información, lo que traduce la importancia que dio a estos componentes de su narración y la prolijidad con que buscó las referencias. Además tal como WWC mencionó en el epígrafe que acompaña este artículo, cada vez que incluía en sus libros algún aspecto médico o de otra ciencia, antes de enviarlos a publicar, los sometía a revisión por especialistas en el tópico respectivo ${ }^{12}$. En muchas de sus obras, los médicos tienen un rol destacado y es llamativo el conocimiento que WWC tenía del carácter de ellos; predomina la visión irónica, como por ejemplo su afirmación que: "El médico que no es lo suficientemente honesto para confesar cuando está desconcertado es un miembro habitual de la profesión médica en todos los países"”.

Es sorprendente la verosimilitud de sus descripciones, lo que hace imposible no pensar que pudo tener contacto directo con diversas patologías. En la novela "El juego del escondite" (1854), WWC reconoce una exhaustiva preparación en la literatura médica de la época sobre la pérdida de los sentidos, como el estudio de la obra "La pérdida de los sentidos" del Dr. Ditto ${ }^{13}$, pero también es obvio que le fue muy valioso su agudo poder de observación. En "La piedra lunar", menciona 
como fuente de sus lecturas la obra "Fisiología Humana” del Dr. Elliotson, conocido fisiólogo de la época ${ }^{11}$.

En “Corazón y Ciencia” (1883), hace una aguda crítica de la vivisección (disección de animales vivos con el fin de investigación) muy en boga en esa época a raíz de los trabajos del destacado neurólogo escocés David Ferrier, quien fue cuestionado por ellos, incluso legalmente ${ }^{14}$. El cuestionamiento surgió de los experimentos de estimulación eléctrica cortical cerebral una vez que los animales se recuperaban de la anestesia. WWC preparó su libro en gran parte basado en la obra de Ferrier "The localization of cerebral disease" $(1878)^{14}$. WWC criticó estos estudios especialmente, porque según él, muchos de ellos eran sólo para justificar el ego de algunos investigadores siendo que eran muy pocos quienes lograban un verdadero aporte científico ya que "la mayoría duda aún de sus propias conclusiones, lo que no les da derecho para seguir matando perros ni gatos"14. Sin embargo, debemos reconocer que los estudios de Ferrier fueron pioneros y muy importantes en el estudio de las funciones motoras y sensitivas cerebrales y la fisiopatología de la epilepsia ${ }^{14}$.

WWC nos revela en la mayoría de sus obras, un sorprendente espíritu científico, no sólo en el contenido, sino en la forma misma de preparar sus escritos literarios. Conocer a este genial escritor depara no sólo interiorizarse en sorprendentes relatos, sino también encontrar admirables e inesperadas descripciones médicas que valoran aún más su obra.

Agradecimientos: Al Dr. Peter Wolf, profesor del Centro Danés para Epilepsia, por sus comentarios y referencias sobre los aportes en epilepsia de la obra de WWC; a Andrew Gasson y Paul Lewis, directores de The Wilkie Collins Society de Londres, por su gentileza en proporcionar información, al Profesor Andrew Lees, del Instituto de Estudios Neurológicos Reta Lila Weston, University College de Londres, por las referencias sobre los estudios de David Ferrier. Igualmente a Osvaldo Gil, bibliotecario de la Facultad de Medicina, Universidad de Chile, por su apoyo en las referencias bibliográficas.

\section{Referencias}

1. Gasson A. Wilkie Collins: an illustrated Guide. Oxford University Press, London. 1998.

2. http://www.web40571.clarahost.co.uk/wilkie/wilkie.htm. Reproducciones con permiso de Paul Lewis, creador del sitio.

3. www.wilkie-collins.info. Acceso el 10 enero 2011.

4. Borges JL. Mi biblioteca personal. Alianza Editorial, Madrid. España. 1988.

5. Eliot TS. Dickens and Collins en: Selected Essays. Houghton Mifflin Harcourt Eds. New York 1950-412.

6. Clarke WW. La vida secreta de Wilkie Collins. Editorial Alba, Barcelona 1999.

7. Wolf P. Epilepsy and Catalepsy in Anglo-American Literature Between Romanticism and Realism: Tennyson, Poe, Eliot and Collins. J Hist Neurosci 2000; 9: 286-93.

8. Wilkie Collins. Pobre Señorita Finch. Editorial Alba. Madrid 1998.

9. Miranda M, Williams AM, García-Borreguero D. Thomas de Quincey and his restless legs syndrome and depicted in his Confessions of an English opium-eater. Mov Disord 2010; 13: 2006-2009.

10. Wilkie Collins. La Piedra Lunar. Montesinos Editores. 1998.

11. Seagal S. Wilkie Collins: Victorian novelist as psychopharmacologist. Hist Med Allied Sci 1983; 38: 16175.

12. Wilkie Collins. Armadale. Ediciones Bolsillo, Barcelona 1998.

13. Wilkie Collins. El juego del Escondite. Montesinos Editores. 2010.

14. Otis L. Howled out of the country: Wilkie Collins and H.G. Wells retry David Ferrier en: Neurology and Literature, 1860-1920 (Palgrave Studies in Nineteenth-Century Writing and Culture). Editor. Anne Stiles, Palgrave Macmillan.Londres 2007; 27-51. 